\title{
Inconsistências e consequências da variável raça para a mensuração de desigualdades
}

\section{Inconsistencies and consequences of the race variable for the measurement of inequalities}

\author{
Jeronimo Oliveira Muniz*
}

\begin{abstract}
Resumo: "Não é possível separar as pessoas com base na raça no Brasil" (Fry et al., 2007). Esse argumento é parte do sistema de classificação racial brasileiro pelo menos desde o começo do século 20, tendo sido também uma razão para ceticismo quanto ao sucesso dos programas de ação afirmativa implementados para mitigar as desigualdades raciais do Brasil. Este artigo investiga a influência da inconsistência da classificação racial sobre o nível e composição das desigualdades educacionais e de consumo. A partir dos dados da Pesquisa Social Brasileira (PESB) de 2002 demonstra-se que os indivíduos que têm sua raça/cor inconsistentemente classificada respondem por pelo menos metade da desigualdade total e por mais de um terço da desigualdade inter-racial. Quando os inconsistentemente classificados são excluídos da análise, entretanto, a proporção das desigualdades inter-raciais não se altera substantivamente, demonstrando que a mesma é fracamente influenciada pela incerteza racial atrelada ao método de coleta utilizado.

Palavras-chave: Escolaridade. Cor. Consumo. Desigualdade. Distribuição racial.
\end{abstract}

Abstract: "It is not possible to separate people by race in Brazil" (Fry et al., 2007). This common and long-standing argument is part of the Brazilian racial classification system at least since the early 20 th century. The inability to identify target populations has also been a reason to be skeptical about the success of affirmative action programs implemented to mitigate persistent racial inequalities in Brazil. This article investigates the association between inconsistencies in racial classification and the level and composition of inequalities in education and consumption. Using unique data from the 2002 Brazilian Social Survey (PESB), we show that individuals whose race/color was inconsistently reported account for at least half of total inequality and more than a third of inter-racial inequality. After excluding inconsistently classified individuals from our sample, however, the share of inter-racial inequalities does not substantially change, demonstrating that racial uncertainty has little influence on racial inequality in Brazil.

Keywords: Schooling. Skin color. Consumption. Inequality. Racial distribution.

\footnotetext{
* Doutor em Sociologia/Demografia pela University of Wisconsin, Madison, EUA, professor no PPG em Sociologia da Universidade Federal de Minas Gerais (UFMG), diretor do Centro de Pesquisas Quantitativas em Ciências Sociais (CPEQS/UFMG) e coordenador do Programa de Treinamento Intensivo em Metodologia Quantitativa (MQ) da UFMG em Belo Horizonte, Minas Gerais, Brasil<jeronimo@fafiich.ufmg.br>.

Esta pesquisa foi desenvolvida com o apoio de Bolsa de Produtividade em Pesquisa do Conselho Nacional de Desenvolvimento Científico e Tecnológico, processo 307375/2014-5. O autor agradece aos pareceristas anônimos pelas sugestões.
} 


\section{Síntese do pensamento racial brasileiro do século 20 e 21}

A classificação racial do povo brasileiro é motivo de polêmica desde os primeiros levantamentos censitários. Entre 1900 e 1930 os censos demográficos não coletaram a informação racial por duas razões: por causa da influência das elites; e também sob o pretexto de ela ser subjetiva, imprecisa e "não científica" (Loveman, 2009). Estas características tornavam a variável raça incompatível com os valores almejados pela então Diretoria Geral de Estatística, cuja meta era produzir conhecimentos estatísticos objetivos para guiar ações governamentais para o progresso da nação. Ainda que parte da intelectualidade de época acreditasse que "branquitude" e progresso caminhariam juntos - e ainda que houvesse evidência para crer que caso a pergunta sobre raça fosse incluída nos censos ela tenderia a inflar o número de brancos ${ }^{1}-$, durante as três primeiras décadas do século passado a informação racial não constou nos registros estatísticos oficias da nação. A ausência da raça nos registros estatísticos do começo do século passado deveu-se não só a uma tentativa de aderir a padrões censitários internacionais, que primavam por uma suposta objetividade das informações coletadas, mas também se especula que restrições orçamentárias impostas ao Censo, assim como a influência das elites da época, que desejavam diminuir a importância da composição racial do Brasil, tenham contribuído para a omissão da raça durante o período (Telles, 2004, p. 31).

Já em 1940 constavam no questionário do Censo apenas as opções branca, preta e amarela. "Outras respostas" foram codificadas como de cor parda, opção que não foi incluída graças ao consenso da época de que no Brasil não se poderia falar em raça já que aqui esse conceito não seria "nativo" e nem óbvio e pragmático como no caso norte-americano (Guimarães, 2003). Não havia razão para se mensurar a cor da população já que aqui éramos todos iguais e representávamos uma mítica "democracia racial" marcada pela ausência de barreiras raciais à ascensão social. Gilberto Freyre (1987 [1933]), Pierson (1945) e alguns dos estudos patrocinados pela Unesco como Azevedo (1966, 1996), Harris (1952) e Wagley (1952) disseminaram no pensamento racial brasileiro da primeira metade do século passado a ideia de que aqui seria uma democracia racial, corroborada pela miscigenação e exaltada pelo amorenamento (Osorio, 2008). O projeto da Unesco visava "estimular a produção de conhecimento científico a respeito do racismo, abordando as

\footnotetext{
${ }^{1}$ Supôs-se que haveria aumento populacional dos brancos por conta da inexatidão classificatória dos mulatos, que "dar-se-iam por brancos" nos formulários do Censo, e também por conta dos fluxos imigratórios da Europa verificados durante o período. Tais argumentos são apresentados por Oliveira Viana (1926, apud Loveman, 2009, p. 462-463, nas notas de rodapé 74 a 76).
} 
motivações, os efeitos e as possíveis formas de superação do fenômeno" (Maio, 1999, p. 143).

Oracy Nogueira (1985; 1998), Florestan Fernandes $(1965 ; 2007)$ e alguns de seus ex-alunos (Ianni, 1962, 1987; Cardoso, 2000, 2003; Cardoso e Ianni, 1960) representam a segunda vertente do pensamento racial do Brasil. ${ }^{2}$ Nessa linha teórica, ao contrário da anterior, o Brasil seria sim um país racista, mas a discriminação vigente desapareceria na medida em que o país fosse se modernizando a partir da industrialização e da racionalização crescente do mundo. Com o desenvolvimento do capitalismo uma sociedade de classes se imporia sobre a questão racial na medida em que a educação e a inserção no mercado de trabalho fossem se universalizando. O otimismo de Fernandes quanto às benesses do capitalismo como mitigador de desigualdades raciais, entretanto, mostrou-se exagerado já que a distância entre classes, ao invés de ser abolida, foi na verdade reforçada com o passar dos anos.

A terceira onda teórica, dos anos 70 em diante, é encabeçada por Carlos Hasenbalg (2005 [1979]) e Nelson do Valle Silva (1978; 1979; 1980), que utilizaram métodos quantitativos para mostrar que à trajetória de vida dos negros se atrelam desvantagens cumulativas que só podem ser reduzidas a partir de ativismos políticos e ações afirmativas específicas (Osorio, 2009; Ribeiro, 2006). Segundo Munanga (1999) e Hasenbalg - em entrevista concedida a Guimarães (2006) -, somente a partir da intervenção estatal e da politização da questão racial, a partir da criação de uma identidade racial bipolar (brancos e não-brancos), é que haveria condições políticas para superar a ideologia coletiva da ausência de conflito racial e a consequente desmobilização política dos negros. A segunda e a terceira ondas teóricas negam a tese Freyriana e são, portanto, antíteses da mesma.

Já no século 21, Telles (2003) e Moraes Silva (2015) tentaram conciliar os estudos anteriores sugerindo que, no contexto brasileiro, desigualdades raciais podem existir sem racismo. Por um lado, relações horizontais de sociabilidade inter-racial (uniões heterogâmicas, baixa segregação, cultura compartilhada e branda convivência social) seriam compatíveis com o ideal de democracia racial sugerido por Freyre. Nesta perspectiva, a boa convivência inter-racial caracterizaria a fragilidade das fronteiras raciais simbólicas entre brancos e negros, que não se perceberiam como grupos bipolares isolados, mas sim como parte de uma identidade coletiva comum. Por outro lado, e

\footnotetext{
${ }^{2}$ Os estudos de Oracy Nogueira e Florestan Fernandes também são parte integrante ou desdobramentos do projeto da Unesco para o sul do país, e refletem em alguma medida "a difícil e tensa relação entre racismo e mito da democracia racial brasileira. Essa tensão permanecerá ao longo do Projeto Unesco" (Maio, 2009, p. 152).
} 
simultaneamente, haveria fronteiras sociais evidentes entre grupos raciais verticalmente separados a partir de hierarquias de poder socioeconômico. Estas fronteiras foram constatadas por Oracy Nogueira, Florestan Fernandes e Carlos Hasenbalg a partir de medidas persistentes de desigualdade racial. A especificidade do caso brasileiro, portanto, ao invés de restringir-se à democracia racial ou ao racismo explícito, estaria na possibilidade de coexistência e interação entre as dimensões de assimilação horizontal e desigualdade racial vertical (Telles, 2003), ou entre as fronteiras simbólicas e sociais (Moraes Silva, 2015). ${ }^{3}$ Supõe-se que os protagonistas da negociação de fronteiras seriam sobretudo os pardos, pela sua habilidade de fluir livremente entre as duas polaridades de cor sem ser estigmatizados. Ao atravessarem as fronteiras simbólicas, entretanto, os pardos alterariam também as fronteiras sociais ao levarem consigo atributos socioeconômicos capazes de diluir ou concentrar desigualdades raciais previamente observadas. Entender empiricamente como a incerteza racial classificatória afeta a desigualdade é, portanto, crucial para compreendermos as consequências que fronteiras raciais e simbólicas fracas (ex. volatilidade racial) têm sobre fronteiras sociais fortes (ex. desigualdade racial). É esta a proposta deste artigo.

\section{Sobre a conexão entre a mensuração da raça, ações afirmativas e desigualdades}

O debate acerca das cotas raciais levantou a questão de que para se viabilizar políticas públicas seria preciso se ter clareza sobre quem representaria o público alvo (Feres Júnior, 2007; Muniz, 2010). Afinal, sendo a raça - o critério distributivo de políticas - variável, as populações alcançadas também seriam. A incerteza classificatória por cor seria assim uma das razões para não se adotar cotas raciais como critério de inclusão nas instituições de ensino superior no Brasil sob a dificuldade de identificação dos beneficiários a partir de critérios objetivos de distinção além da autodeclaração.

\footnotetext{
${ }^{3}$ Cabe agregar que Silva e Hasenbalg (1999) já haviam identificado estas duas dimensões, que Telles chamou de vertical e horizontal, anteriormente. Em entrevista concedida a Antônio Sérgio Guimarães, Carlos Hasenbalg lembra que: “[...] quando estudamos essas desigualdades, opondo brancos/não-brancos (pretos e pardos), nos referimos estritamente a processos de estratificação socioeconômica. Quando examinamos outras dimensões da vida social envolvendo a sociabilidade dos indivíduos (por exemplo, o casamento e a amizade), esse padrão não se verifica, os pardos se diferenciando dos pretos e se aproximando mais dos brancos. Poder-se-ia dizer que há uma disjunção aparente entre o processo de estratificação, que diz respeito ao funcionamento das principais instituições (escola e mercado de trabalho), e a vida social dos indivíduos, caracterizada por barreiras mais fluidas e ambíguas. Essa disjunção tende a corresponder à distinção feita por Sansone (1993) entre as "áreas duras" e as "áreas moles" das relações raciais no Brasil" (Guimarães, 2006).
} 
Ao sistematizar os argumentos de eminentes cientistas sociais contra as cotas raciais, contidos na segunda parte da antologia Divisões perigosas: políticas raciais no Brasil contemporâneo (Fry et al., 2007), Feres Júnior (2008, p. 58) constatou que cerca de um terço dos 50 textos publicados no livro manifestavam contra as cotas o argumento de "não ser possível separar as pessoas com base na raça no Brasil". A dificuldade de se adotar procedimentos classificatórios objetivos foi o sétimo argumento mais prevalente entre os vinte e dois motivos mobilizados pelos autores contra as cotas raciais.

A dificuldade de identificação dos beneficiários também se torna evidente ao examinarmos a literatura relacionada. Por um lado, pode-se utilizar o argumento de que os sujeitos de direitos, geneticamente falando, corresponderiam a $87 \%$ das pessoas que são descendentes de africanos (Pena; Bortolini, 2004). Isso corresponderia a 166 milhões de pessoas pelo censo de 2010. Por outro lado, o percentual de pessoas que se autodeclaram como pretas $(7,6 \%)$ ou pardas $(43,1 \%)$ corresponde a $50,7 \%$. Além disso, há evidências demonstrando a ambiguidade das medidas de raça ou cor no Brasil quando se utilizam diferentes estratégias de coleta da informação racial (Bailey; Loveman; Muniz, 2013; Bailey; Telles, 2006; IBGE, 2011; Loveman; Muniz; Bailey, 2012; Simões; Jeronymo, 2007; Telles; Lim, 1998). Muniz (2012), por exemplo, demonstra que somente metade dos entrevistados em uma pesquisa de representatividade nacional se classifica ou é classificada dentro da mesma categoria racial quando se adota quatro metodologias distintas de coleta da informação racial. A inconsistência classificatória racial, a dificuldade de se identificar beneficiários legítimos das cotas, seria assim uma das barreiras técnicas à implantação de tais ações afirmativas. Mesmo que esta barreira esteja superada desde 2012, a partir da lei de cotas federal, as polêmicas em torno de fraudes classificatórias ainda continuam. ${ }^{4}$

Além disso, seria possível argumentar que, diante da incerteza de cor - em função de "fraudes" de declaração ou diferentes métodos de classificação -, a desigualdade racial também poderia ser variável. Como medidas de desigualdade dependem de como os recursos são distribuídos entre pessoas pertencentes a diferentes grupos raciais, elas podem se alterar: 1) diante de uma redistribuição de recursos entre grupos fixos; 2) diante de uma redistribuição de grupos (ex. reclassificação racial) entre recursos; ou 3) ambos. No caso brasileiro, como a raça é uma variável volátil, o segundo

\footnotetext{
${ }^{4}$ Ver por exemplo reportagem recente da Folha de São Paulo: "Falsos cotistas viram alvo de universitários negros pelo país", publicada no dia 9 de abril, 2016. Disponível em: <http://folha. com/no1759326>.
} 
tipo de dinâmica entra em vigor, fazendo com que a desigualdade racial seja causa, mas não necessariamente consequência exclusiva, das políticas de ação afirmativa.

Se o modo de classificação da cor alterar as medidas de desigualdade, que são motivação para políticas de inclusão, a lógica e razão das ações afirmativas estariam comprometidas. Por outro lado, caso as desigualdades sejam insensíveis à incerteza classificatória, haverá evidência para se refutar o argumento de que as dificuldades de identificação racial devam ser um empecilho à adoção de políticas de inclusão racial, já que neste caso a motivação e o instrumento de avaliação das políticas (a própria desigualdade racial) estaria preservado da volatilidade taxonômica.

Diante do cenário de incerteza classificatória, e tendo em vista que um dos principais argumentos a favor das cotas pauta-se na "correção de injustiças e desigualdades raciais" (Campos, 2012), cabe então perguntar qual seria o impacto desta incerteza sobre as desigualdades mensuradas na população e entre grupos raciais pré-definidos a partir das categorias assumidas pelo IBGE. Sendo a cor uma variável volátil, arbitrária e sensível ao método de classificação utilizado, seria ela influente sobre medidas de desigualdade, tanto na população quanto entre grupos raciais consistentemente classificados? Se as desigualdades raciais forem sensíveis à volatilidade classificatória, isto é, se as medidas de desigualdades se alterarem em função da inclusão ou exclusão dos racialmente inconsistentes, o instrumento alvo de avaliação da política (as desigualdades) estaria comprometido pelo próprio critério (a raça) adotado para sua implantação. Alternativamente, caso as desigualdades raciais sejam insensíveis às inconsistências classificatórias, haveria suporte para não se rejeitar a raça como um critério de inclusão social já que esta, mesmo sendo incerta e volátil, não comprometeria o objeto e instrumento fim de mensuração da eficácia da política, ou seja, o próprio tamanho das desigualdades.

A influência da variabilidade taxonômica sobre a composição racial populacional e as consequências de se adotar tipologias raciais binárias sobre a desigualdade de renda foram examinadas em artigos recentes (Bailey; Loveman; Muniz, 2013; Loveman; Muniz; Bailey, 2012; Muniz, 2012), mas a influência da inconsistência racial sobre medidas de desigualdade educacional e de consumo ainda não foi analisada. Ainda não há evidência sobre a hipótese de que a incerteza racial classificatória seja um argumento pertinente para a não utilização da variável como critério locativo e distributivo de benefícios mitigadores de desigualdades. Como decorrência, o argumento contra as cotas raciais pautado na inabilidade de mensuração confiável da raça tem se 
mantido como "pura especulação sem base em qualquer evidência sólida" (Feres Júnior, 2008, p. 71).

Neste artigo investigamos a influência da inconsistência classificatória em duas dimensões da desigualdade: anos de estudo e consumo. Utiliza-se a escolaridade por ela ser um indicador amplamente disponível e aceitável de bem-estar e status econômico atrelado às trajetórias de vida e mobilidade social individual. Além disso, o diferencial de escolaridade entre brancos e negros é comumente citado como motivo para adoção de políticas compensatórias. $\mathrm{O}$ consumo de bens duráveis, por sua vez, revela uma dimensão mais permanente da desigualdade por ser uma medida de estoque e não de fluxo econômico. A posse de bens de consumo duráveis é relevante por ter efeito ordinal e monotônico sobre o bem-estar (ex. quanto mais bens melhor), por ter o potencial de aumentar a renda dos que se encontram em situação de pobreza (ex. carro, fogão e geladeira podem ser utilizados para a produção e distribuição de bens comercializáveis), e por propiciar a estes a possibilidade de lidar com choques adversos de renda (ex. desemprego e gastos de saúde inesperados) (Neri et al., 1999). Vários estudos têm investigado a pobreza e a desigualdade da perspectiva do consumo (Rocha, 1988, 2000; Reddy, Pogge, 2002; Ferreira, Lanjouw, Neri, 2001), incluindo estudos voltados para a desigualdade racial (Oliver, Shapiro, 2006), sob a justificativa de que as desigualdades observadas nestas duas dimensões, escolaridade e consumo, diferem substancialmente apesar de estarem correlacionadas.

Ao examinarmos o impacto da já conhecida inconsistência classificatória racial (Muniz, 2012) sobre medidas tradicionais de desigualdade educacional e consumo, testaremos a influência que a ambiguidade racial brasileira tem sobre a mensuração de desigualdades. Para orientar o debate formulamos as seguintes perguntas: 1) qual a parcela da desigualdade educacional e de consumo que se deve ao grupo dos brancos, pretos e pardos quando se utiliza a autodeclaração racial, tal como feito pelo IBGE? 2) como estas desigualdades se alteram quando consideramos apenas aqueles cuja raça/cor é consistentemente classificada em pelo menos três metodologias taxonômicas distintas? 3) qual o impacto da inconsistência classificatória sobre as desigualdades inter-raciais? E sobre a desigualdade total?

A seção seguinte descreve o banco de dados. A quarta seção apresenta a metodologia empregada para decompor as desigualdades educacionais e de consumo entre subgrupos raciais. A quinta seção apresenta os resultados da decomposição e discute suas implicações para a construção de desigualdades. A última seção conclui, enfatizando as consequências da variável raça para a mensuração de desigualdades. 


\section{Dados}

A Pesquisa Social Brasileira (PESB) realizou 2.364 entrevistas domiciliares em todo o Brasil durante o ano de 2002 (Almeida; Schroeder; Cheibub, 2004). Foram pesquisados valores e atitudes relacionados ao: "Jeitinho brasileiro" (Da Matta, 1988), à violência e criminalidade, relativos às relações raciais, à sexualidade e visões de mundo relacionadas à intervenção do estado na economia. Tal amostra é representativa da população adulta brasileira (a partir de 18 anos) para as cinco regiões do país (Norte, Nordeste, Centro-Oeste, Sudeste e Sul).

Escolheu-se tal pesquisa por ela servir como marco referencial anterior à adoção nacional massiva de políticas de inclusão racial, coincidindo apenas com o período de início das primeiras experiências de ação afirmativa no Brasil (ex. cotas raciais na Uerj e Uenf em 2001), e também pela sua abordagem singular sobre questões raciais. A pesquisa permite conhecer a composição racial brasileira e averiguar a consistência entre diferentes metodologias de coleta da informação sobre raça e cor, indo assim além da declaração do entrevistado. Através das informações coletadas pela PESB pode-se medir a raça/cor através da autoclassificação (entrevistado declara qual a sua raça), heteroclassificação (o entrevistador, devidamente treinado, assinala a raça do entrevistado), hipodescendência (a raça dos entrevistados passa a ser igual à do ascendente direto mais escuro) e foto-classificação (entrevistadores utilizamse da percepção dos usuários sobre a raça de pessoas apresentadas em oito fotos para assinalar a raça do entrevistado).

Tendo em vista que a PESB utiliza essas quatro metodologias aplicadas às mesmas pessoas, se pode cruzar as informações para averiguar a consistência racial e responder à pergunta aqui apresentada: O modo de coleta da variável raça influencia nossas percepções sobre as diferenças observadas entre grupos raciais? Com esta disposição do banco de dados podemos separar os grupos raciais segundo a metodologia de coleta adotada pelo IBGE, e compará-los aos brancos, pretos e pardos consistentemente classificados nos outros métodos taxonômicos adotados pela PESB. Para isso dividimos os consistentemente classificados em dois grupos. O primeiro inclui todos os que se classificaram ou foram classificados dentro da mesma categoria racial nas quatro metodologias taxonômicas empregadas, incluindo a metodologia de classificação baseada na cor do ascendente mais escuro. O segundo grupo considera como consistentemente classificado todos que tiveram a mesma raça/cor em três metodologias, excluindo aquela baseada na cor dos pais. Em ambos os casos, o grupo dos inconsistentes passa então a representar 
uma terceira categoria classificatória, além da dos brancos, pardos e pretos. Em suma, trata-se de comparar o modo como o IBGE avalia a raça hoje com casos racialmente consistentes nas outras três metodologias apresentadas, utilizando técnicas de decomposição de desigualdades. O gráfico 1 ilustra a distribuição dos racialmente inconsistentes em quatro metodologias, e tendo como referência a distribuição racial do IBGE (no eixo horizontal).

Gráfico 1. Cruzamento entre categorias raciais do IBGE e categorias raciais consistentes em 4 metodologias

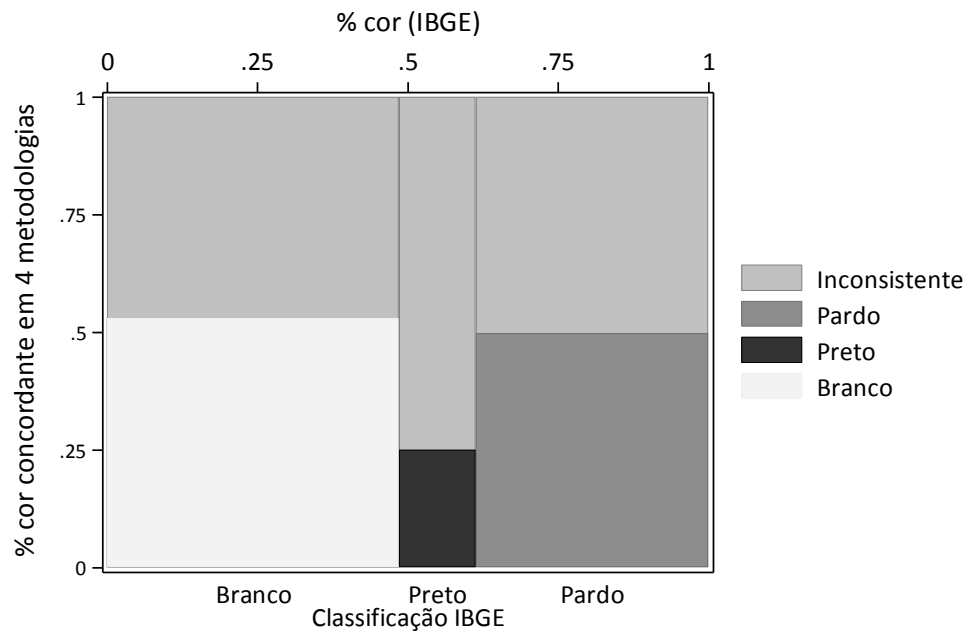

Fonte: Elaboração própria a partir dos dados da PESB2002.

O gráfico 1 mostra que cerca de metade dos brancos e dos pardos classificados de acordo com as categorias do IBGE não mantêm essa mesma classificação quando sua raça é captada a partir de outras metodologias (cor dos pais, percepção do entrevistador, fotografias). No caso dos pretos, o percentual de inconsistentes é ainda maior, quase igual a 75\%. Os autodeclarados pretos são os que possuem as maiores chances de serem inconsistentes na sua classificação racial. ${ }^{5}$ Há, portanto, uma parcela considerável de pessoas cuja cor não se mantém a mesma quando esta informação é captada de outras formas.

\footnotetext{
$541 \%$ dos racialmente inconsistentes em pelo menos três metodologias de classificação se autodenominam pardos. Esse percentual diminui para 37\% quando utilizamos o critério de inconsistência baseado em quatro metodologias. Os autodenominados pretos são os que possuem o maior percentual de pessoas racialmente inconsistentes: $59 \%$ deles quando três metodologias são utilizadas, e $75 \%$ dos pretos são inconsistentemente classificados quando quatro metodologias são utilizadas como critério de inconsistência.
} 
Conhecer o impacto que os racialmente inconsistentes têm sobre medidas de desigualdade é o principal objetivo deste artigo. Se a incerteza racial classificatória não alterar substantivamente as medidas de desigualdade, isto é, se ela se mostrar inócua diante do que se quer combater, haverá evidência para se descartar o argumento da incerteza racial como empecilho à adoção das ações afirmativas. A amostra analítica possui informações válidas e diferentes de zero de escolaridade, consumo e cor para 2039 casos, o que corresponde a quase $90 \%$ do total de entrevistas realizadas pela PESB.

\section{Metodologia}

\section{Medidas de escolaridade e consumo}

Adotou-se o número de anos de estudo dos indivíduos como medida de escolaridade por esta informação estar disponível para quase todos os entrevistados do banco de dados. A escolaridade mínima é igual a 1, para pessoas analfabetas, e igual a 20 para pessoas com doutorado completo. Já o padrão de consumo é mensurado a partir de um índice construído segundo a posse ou a presença de bens duráveis e serviços no domicílio. O índice de consumo é definido segundo a equação

$$
\sum_{i=1}^{n} \frac{D i . n}{\overline{D i}}
$$

sendo $D i$ igual a 1 quando o domićlio possui o bem durável ou serviço $i$ (rádio, banheiro, empregada, aspirador de pó, máquina de lavar, videocassete, geladeira, congelador, telefone, telefone celular, computador, carro, internet); e 0 se o domicílio não possui o bem ou serviço $i$. Já $\overline{D i}$ é o percentual de domicílios na PESB com o bem durável $i$; e $n$ é o número absoluto de bens e serviços presentes no domicílio. $\mathrm{O}$ índice é padronizado para variar entre 0 e 1 , sendo 1 o valor máximo de consumo. ${ }^{6}$ Além da escolaridade, usa-se o padrão de consumo relativo ao invés da renda pelo fato dele: 1) ser mais permanente do que a renda declarada; 2) apresentar poucos casos de omissão ou sub-registro; e 3 ) apresentar uma alternativa ao frequente problema da endogeneidade entre cor declarada e padrão de renda. Esse mesmo índice foi utilizado por Muniz (2012) para estudar a associação entre o consumo de bens e serviços e a probabilidade de ser branco ou preto quando se utiliza diferentes metodologias de coleta da

\footnotetext{
${ }^{6}$ Metodologias mais sofisticadas para a construção do índice proposto, tais como análises fatoriais ou de componentes principais, fornecem resultados similares. A distribuição de frequência relativa de tais índices é praticamente a mesma do aqui empregado. Artigos que utilizam tais índices são McKenzie (2005) e Filmer; Pritchett (1999).
} 
informação racial. A tabela abaixo apresenta estatísticas descritivas dos anos de estudo e do índice de consumo da amostra analítica $(n=2039)$ segundo a tipologia de coleta do IBGE e o grau de consistência da informação racial:

Tabela 1. Escolaridade e consumo segundo raça/cor

\begin{tabular}{|c|c|c|c|c|c|c|c|c|c|}
\hline & \multicolumn{3}{|c|}{ IBGE } & \multicolumn{3}{|c|}{$\begin{array}{l}\text { Consistência racial } \\
\text { em } 3 \text { metodologias }\end{array}$} & \multicolumn{3}{|c|}{$\begin{array}{l}\text { Consistência racial } \\
\text { em } 4 \text { metodologias }\end{array}$} \\
\hline & média & d.p. & mediana & média & d.p. & mediana & média & d.p. & mediana \\
\hline \multicolumn{10}{|c|}{ Índice de consumo } \\
\hline Brancos & 0,135 & 0,113 & 0,096 & 0,149 & 0,116 & 0,114 & 0,163 & 0,119 & 0,132 \\
\hline Pretos & 0,061 & 0,059 & 0,043 & 0,068 & 0,070 & 0,050 & 0,070 & 0,077 & 0,039 \\
\hline Pardos & 0,078 & 0,076 & 0,051 & 0,077 & 0,072 & 0,053 & 0,076 & 0,072 & 0,051 \\
\hline Inconsistentes & & & & 0,083 & 0,086 & 0,051 & 0,086 & 0,086 & 0,057 \\
\hline \multicolumn{10}{|c|}{ Índice de consumo } \\
\hline Brancos & 8,006 & 4,599 & 8 & 8,641 & 4,535 & 9 & 8,999 & 4,560 & 10 \\
\hline Pretos & 5,443 & 4,149 & 5 & 5,898 & 4,152 & 5 & 5,977 & 4,192 & 5 \\
\hline Pardos & 6,688 & 4,328 & 6 & 6,846 & 4,043 & 7 & 6,665 & 4,045 & 6 \\
\hline Inconsistentes & & & & 6,201 & 4,515 & 5 & 6,522 & 4,458 & 6 \\
\hline
\end{tabular}

Nota: A distribuição relativa dos grupos é apresentada no anexo $\left(\mathrm{f}_{\mathrm{j}}=\mathrm{n}_{\mathrm{j}} / \mathrm{n}\right)$.

Fonte: Elaboração própria a partir dos dados da PESB2002.

A tabela 1 mostra que a maior variabilidade interna está no grupo dos brancos e inconsistentes. Ela também mostra que as médias e medianas de anos de estudo e consumo dos inconsistentes são similares às dos autodeclarados pardos ou pretos.

\section{Construção e decomposição da desigualdade}

Utiliza-se o índice de Theil-L para estimar os níveis de desigualdade educacional e de consumo. Esta medida satisfaz os requerimentos de um bom índice de desigualdade ${ }^{7}$ e é decomponível em fontes de variação intra e intergrupos (Mookherjee; Shorrocks, 1982). Loveman et al. (2012) o utilizaram para estudar cenários contrafactuais da desigualdade de renda entre brancos e

\footnotetext{
${ }^{7}$ Boas medidas de desigualdade satisfazem cinco axiomas: 1) princípio de transferência de Pigou-Dalton: requer que índices de desigualdade aumentem (ou não diminuam) quando há transferências intergrupos; 2) Independência da Escala: medidas de desigualdade devem ser invariáveis a mudanças proporcionais uniformes (ex. mudança de unidade monetária); 3) Princípio de População: a fusão de populações idênticas não deve afetar a desigualdade; 4) Anonimato: a desigualdade deve ser independente de atributos individuais diferentes que não sejam a própria renda (consumo ou anos de estudo); e 5) "Decomponibilidade": a desigualdade total deve estar consistentemente relacionada às partes que constituem a distribuição de renda (consumo ou anos de estudo), tais como subpopulações definidas segundo características específicas (ex. raça, sexo, idade) (Cowell, 1995).
} 
negros, mas ele também já foi empregado para decompor a desigualdade entre pobres e ricos (Muniz, 2012a), e entre outras características sociodemográficas (Ramos; Vieira, 2000; Ferreira; Leite; Litchfield, 2006). O índice de Theil-L é similar ao índice de $\mathrm{Gini}^{8}$ e tem a seguinte forma:

$$
\text { GE (0) = Índice de Theil-L }=\frac{1}{n} \sum_{i=1}^{n} \ln \frac{\mu}{y_{i}}
$$

Sendo $n$ o número de indivíduos na amostra, $y_{i}$ o valor do índice de consumo ou o número de anos de estudo do indivíduo $i, i \in(1,2, \ldots, \mathrm{n}) \mathrm{e}$ $\mu=1 / n \sum y_{i}$ representa a média aritmética do índice de consumo ou dos anos de estudo.

A decomposição da desigualdade foi realizada para cada um dos três cenários de classificação: (1) considerando-se apenas a metodologia do IBGE, (2) para casos racialmente consistentes em todas as quatro metodologias e (3) para casos consistentes de todas as formas de coleta da informação racial, exceto naquela baseada na cor do ascendente direto mais escuro (hipodescendência). A decomposição permite averiguar o quanto das desigualdades educacionais e de consumo é atribuída às diferenças entre indivíduos pertencentes a distintas categorias raciais e o quanto é atribuído às diferenças entre indivíduos da mesma categoria racial. No primeiro cenário decompomos a desigualdade de consumo e anos de estudo entre brancos, pretos e pardos; e nos dois cenários remanescentes incluímos também aqueles cuja cor foi inconsistentemente classificada em pelo menos uma das quatro ou três metodologias utilizadas. ${ }^{9}$ Algebricamente, a desigualdade total GE(0) pode ser definida pela soma das desigualdades intra (within inequality) e entre grupos (between inequality):

$$
G E(0)=I_{W}^{G E(0)}+I_{B}^{G E(0)}=\sum_{j}^{k} f_{j} G E(0)_{j}+\sum_{j}^{k} f_{j} \ln \left(1 / \lambda_{\mathrm{j}}\right)
$$

$f_{j}$ representa a distribuição proporcional do subgrupo $j$ (brancos, pardos, pretos, racialmente inconsistentes) na população, e $\lambda_{\mathrm{j}}=\mu_{\mathrm{j}} / \mu$ representa a média de anos de estudo (ou consumo) para cada categoria $j$ relativa à média da

\footnotetext{
${ }^{8}$ Apesar de terem níveis distintos, a estrutura da desigualdade apresentada por estas medidas é a mesma (Ferreira; Leite; Litchfield, 2006, p. 7).

${ }_{9}^{9}$ Diferentemente do que vem sido feito por Silva (1985) e Hasenbalg e Silva (2003) desde o final dos anos 70, resolvemos não agrupar as categorias "preto" e "pardo" pelo fato dos testes estatísticos de igualdade de média terem demonstrado que estes dois grupos possuem padrões distintos de consumo e de escolaridade.
} 
população. O segundo termo da equação, desigualdade entre grupos, reflete as diferenças entre médias de consumo ou anos de estudo dos subgrupos. Quando decomposto por categorias raciais, corresponde à escolaridade média relativa ponderada por parcelas da população. A divisão dos dois componentes, $I_{w}{ }^{G E(0)}$ e $I_{B}{ }^{G E(0)}$, pela desigualdade total [GE(0)] representa o peso das desigualdades intra e entre grupos na desigualdade total entre indivíduos.

Os componentes intra e entre grupos $j$ podem também ser rearranjados para representarem a participação relativa de cada categoria (branco, pardo, preto ou inconsistente) na desigualdade total. Reagrupando-se os componentes da equação (2) tem-se então a contribuição de cada categoria $j$ para a desigualdade total:

$$
G E(0)=\sum_{j}^{k} I_{j}^{G E(0)}=\sum_{j}^{k} f_{j}\left[G E(0)_{j}+\ln \left(1 / \lambda_{j}\right)\right]
$$

Esta é a estratégia metodológica adotada para inferirmos a importância dos grupos raciais e dos racialmente inconsistentes nas desigualdades total e inter-racial. Os componentes das equações de decomposição das desigualdades educacionais e de consumo encontram-se, respectivamente, nos anexos 1 e 2.

\section{Discussão dos resultados}

\section{Decomposição da desigualdade total}

Ao calcularmos o índice de Theil-L para a amostra em questão percebemos que: 1$)$ a desigualdade educacional $(0,23)$ é menor que a de consumo $(0,43)$; e 2$)$ os casos racialmente inconsistentes compõem mais da metade das desigualdades totais de consumo e anos de estudo. Os percentuais do gráfico 2 representam a contribuição relativa de cada grupo racial para a desigualdade total, e a sua soma totaliza $100 \%$. Baseando-se na equação (3), o gráfico mostra que $74 \%$ da desigualdade de consumo e $77 \%$ da de escolaridade deve-se ao grupo daqueles cuja cor não foi a mesma nas quatro metodologias utilizadas pela PESB. Ao desconsiderar-se a regra baseada na cor do ascendente direto mais escuro, a parcela da desigualdade de consumo atrelada aos casos inconsistentes cai para $58 \%$ e a de anos de estudo para $67 \%$. Mais da metade da desigualdade entre indivíduos, portanto, deve-se ao grupo dos inconsistentes.

A proporção dos racialmente inconsistentes na desigualdade total é grande pois estes correspondem a $37 \%$ ou $52 \%$ da amostra analisada, dependendo do critério de consistência utilizado. ${ }^{10}$

\footnotetext{
${ }^{10}$ Ver a nota 5.
} 
Gráfico 2. Decomposição das desigualdades educacionais e de consumo por grupos raciais

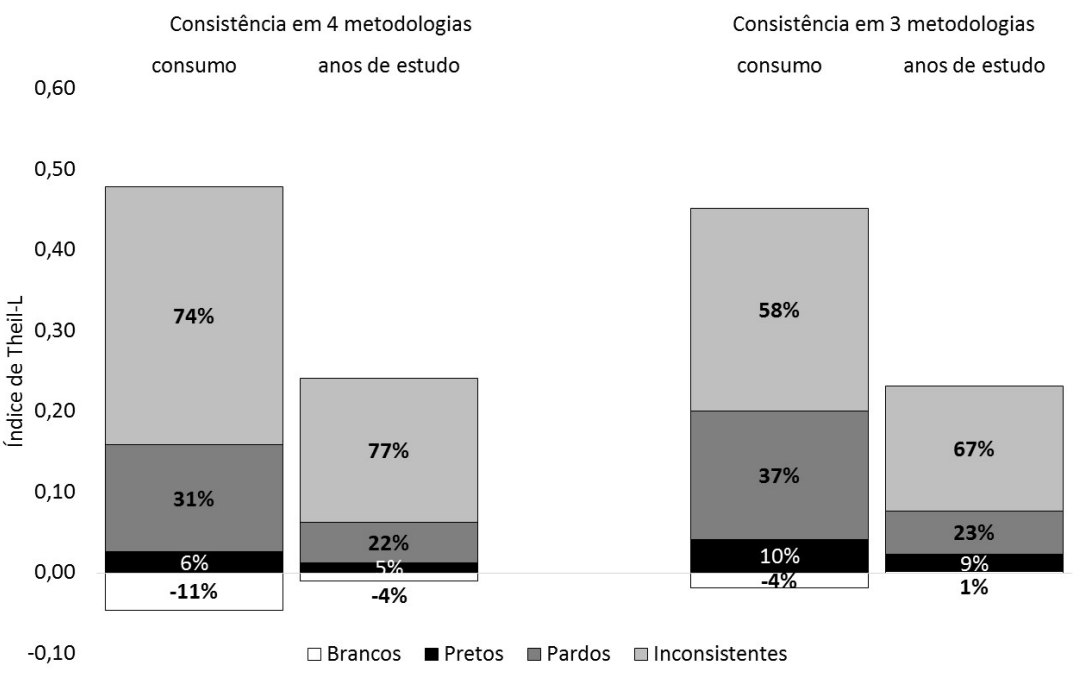

Nota: Os percentuais de cada categoria correspondem à razão $f_{j}\left[\left(G E(0)_{j}+\ln \left(1 / \lambda_{\mathrm{j}}\right)\right] / G E(0)\right.$. Fonte: Elaboração própria a partir dos dados da PESB2002.

\section{Decomposição da desigualdade inter-racial}

O componente inter-racial da desigualdade educacional corresponde a $4 \%$ da desigualdade total, enquanto no caso do consumo este componente representa $12 \%$ da desigualdade. Estes percentuais podem ser entendidos como parcelas das barras apresentadas no gráfico 2, e os percentuais remanescentes destas desigualdades (96\% e $88 \%$ ) representam a desigualdade dentro dos próprios grupos raciais utilizados na decomposição.

Ainda que a desigualdade inter-racial represente apenas a menor parte das desigualdades totais entre pessoas, é sob a justificativa de reduzi-la que políticas de ação afirmativa foram propostas e implementadas. Cabe, portanto, entender qual o peso de cada grupo dentro deste tipo de desigualdade. Isso pode ser feito combinando-se o tamanho relativo dos grupos a uma medida de distância das médias de anos de estudo e consumo dos grupos. O gráfico 3 apresenta a decomposição da desigualdade inter-racial entre brancos, pretos, pardos e inconsistentes: 
Gráfico 3. Decomposição do componente inter-racial da desigualdade de consumo e anos de estudo

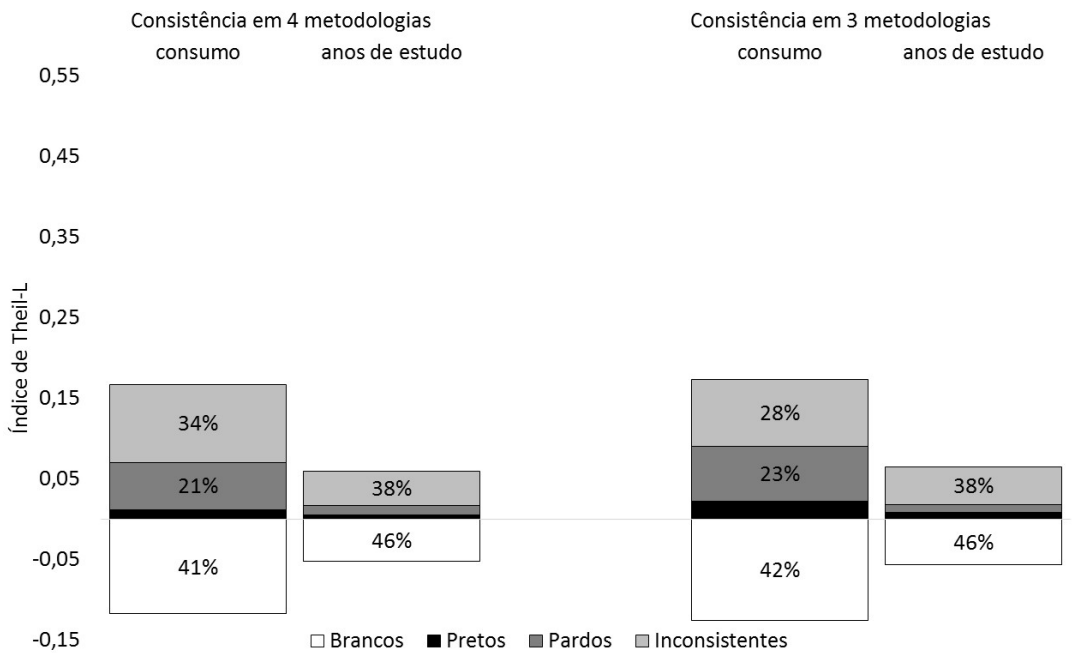

Nota: Os percentuais de cada categoria correspondem à razão $\left|f_{j} \ln \left(1 / \lambda_{\mathrm{j}}\right)\right| \sum_{j}^{k}\left|f_{j} \ln \left(1 / \lambda_{\mathrm{j}}\right)\right|$ Fonte: Elaboração própria a partir dos dados da PESB2002.

O gráfico 3 mostra que a maior parcela da desigualdade inter-racial se deve ao subgrupo dos brancos, que por terem consumo e escolaridade muito acima da média dos grupos, são responsáveis por pelo menos $41 \%$ das desigualdades inter-raciais de consumo e anos de estudo. O gráfico também mostra que o critério de consistência racial, baseado em três ou quatro metodologias, não altera substantivamente a composição da desigualdade inter-racial. Ao desconsiderar a mensuração da raça pela hipodescendência a composição das desigualdades educacionais e de consumo entre os grupos raciais não sofrem alterações significativas. Cerca de um terço das desigualdades entre os grupos raciais, entretanto, concentra-se nos casos inconsistentes. Entre 28\% e 38\% do que percebemos como variação de consumo e anos de estudo entre grupos raciais é função da metodologia empregada para construir a raça/cor de alguém.

Visto que a inconsistência classificatória possui impacto significativo tanto na desigualdade total quanto na inter-racial, é legítimo então perguntar se a desigualdade se alteraria caso as pessoas inconsistentes não fossem consideradas no computo destas medidas. A seção seguinte empreende esta análise contrafactual. 


\section{Inconsistências raciais classificatórias afetam a desigualdade mensurada?}

Para responder a esta pergunta é preciso conhecer qual seria o tamanho das desigualdades educacionais e de consumo se os casos cuja raça foi inconsistentemente classificada fossem excluídos da análise.

Para isso simulamos as desigualdades a partir da exclusão dos racialmente inconsistentes. Excluíram-se 1058 casos (52\%) da amostra analítica que tiveram classificação racial inconsistente em pelo menos uma das quatro metodologias adotadas (auto ou heteroclassificação, fotografia ou hipodescendência). Além disso, para verificar a sensibilidade da "inconsistência" (tornando-a menos restritiva e potencialmente mais "realista"), excluíram-se 758 casos (37\% da amostra analítica inicial de 2039 entrevistas) cuja classificação racial não foi a mesma em três formas de coleta: auto e heteroclassificações a partir das categorias do IBGE, e autodenominação racial a partir de fotografias. As desigualdades resultantes destas seleções amostrais são representadas e decompostas entre grupos raciais no gráfico 4 .

Gráfico 4. Contribuição de brancos, pardos e pretos para a desigualdade educacional total e de consumo segundo a consistência classificatória da raça/cor, Brasil 2002

$\begin{array}{ccccc}\text { anos de } & \text { consumo } & \begin{array}{c}\text { anos de } \\ \text { estudo }\end{array} & \text { consumo } & \begin{array}{l}\text { anos de } \\ \text { estudo }\end{array}\end{array}$

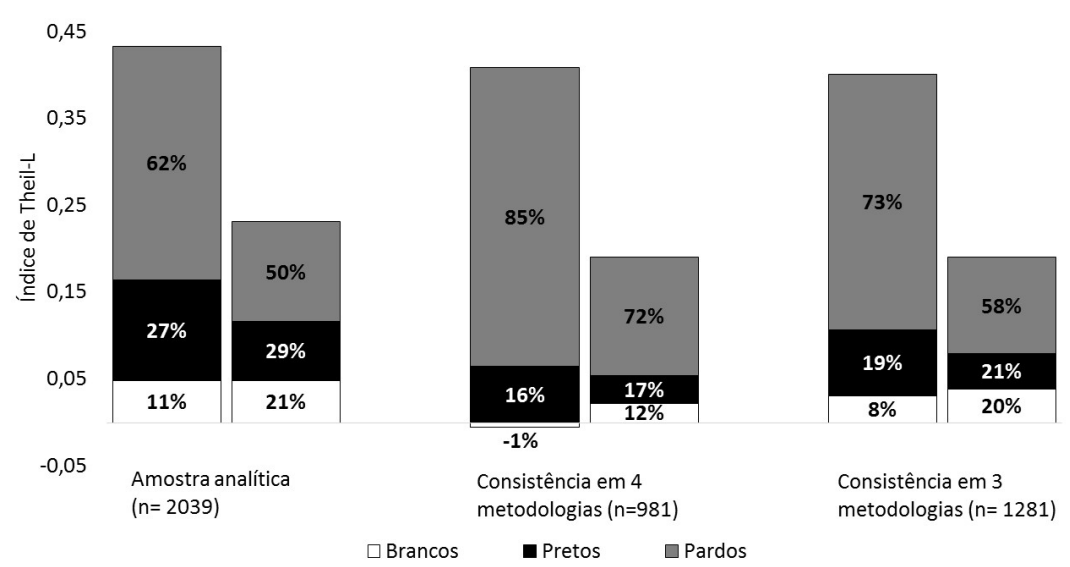

Nota: Os percentuais de cada categoria correspondem à razão $f_{j}\left[\left(G E(0)_{j}+\ln \left(1 / \lambda_{\mathrm{j}}\right)\right] / G E(0)\right.$. Fonte: Elaboração própria a partir dos dados da PESB2002. 
As desigualdades educacionais e de consumo diminuem quando se consideram apenas as pessoas consistentemente classificadas quanto a sua raça/cor. O índice de Theil-L referente aos anos de estudo diminui 17\%, passando de 0,23 para 0,19 ; e o de consumo diminui $7 \%$, passando de 0,43 para $0,40 .{ }^{11} \mathrm{O}$ grupo dos pardos corresponde a maior parcela das desigualdades observadas, sobretudo no que se refere à desigualdade de consumo.

O principal resultado do gráfico 4 é mostrar que há pouca alteração tanto nas desigualdades totais quanto na composição racial das mesmas quando consideramos apenas a amostra daqueles que são consistentemente classificados em pelo menos três metodologias de coleta da informação sobre raça/ cor. A evidência apresentada demonstra, portanto, que a grande quantidade de pessoas racialmente inconsistentes não muda substantivamente o tamanho das desigualdades educacionais e de consumo. A exclusão dos racialmente inconsistentes, entretanto, alteraria a relevância da desigualdade inter-racial na desigualdade total?

O gráfico 5 responde a esta pergunta ao mostrar o impacto dos componentes intra e inter-raciais sobre a desigualdade total. As colunas indicam o percentual da desigualdade total que se deve às diferenças internas aos grupos raciais, e os intervalos acima das colunas referem-se a intervalos estatísticos de $95 \%$ para as desigualdades totais. A diferença entre as estimativas pontuais das desigualdades totais e a altura das colunas representa, portanto, o tamanho do componente inter-racial da desigualdade total. O impacto do componente inter-racial da desigualdade de consumo aumenta de $11 \%$ $(1-0,89)$ para $18 \%(1-0,82)$ quando excluímos da amostra todos os indivíduos cuja raça coletada não foi a mesma em três metodologias taxonômicas. As desigualdades totais, por ouro lado, diminuem ao excluirmos os inconsistentes, mas essa redução é estatisticamente significativa apenas para as desigualdades educacionais.

\footnotetext{
${ }^{11}$ Alternativamente, a redução de $17 \%$ na desigualdade educacional equivale a uma mudança no índice de Gini de 0,32 para 0,29 ; e a uma redução na razão entre percentis extremos de anos de estudo (p90/p10) de 7 para 4,7. Após a exclusão dos casos inconsistentes, os brancos - que antes representavam $48 \%$ da população e detinham $53 \%$ dos anos de estudo - passam a representar $54 \%$ da população e a deter $60 \%$ da distribuição de anos de estudo. Já a redução de $7 \%$ na desigualdade de consumo corresponde a uma mudança no índice de Gini de 0,48 para 0,46 . Como referência, a maior queda da desigualdade de renda brasileira, medida pelo Gini, foi de 0,60 para 0,52 entre 1993 e 2014 .
} 
Gráfico 5. Intervalos de confiança paraa desigualdade total e seus componentes intra e inter-raciais segundo a consistência classificatória da raça/cor, Brasil 2002

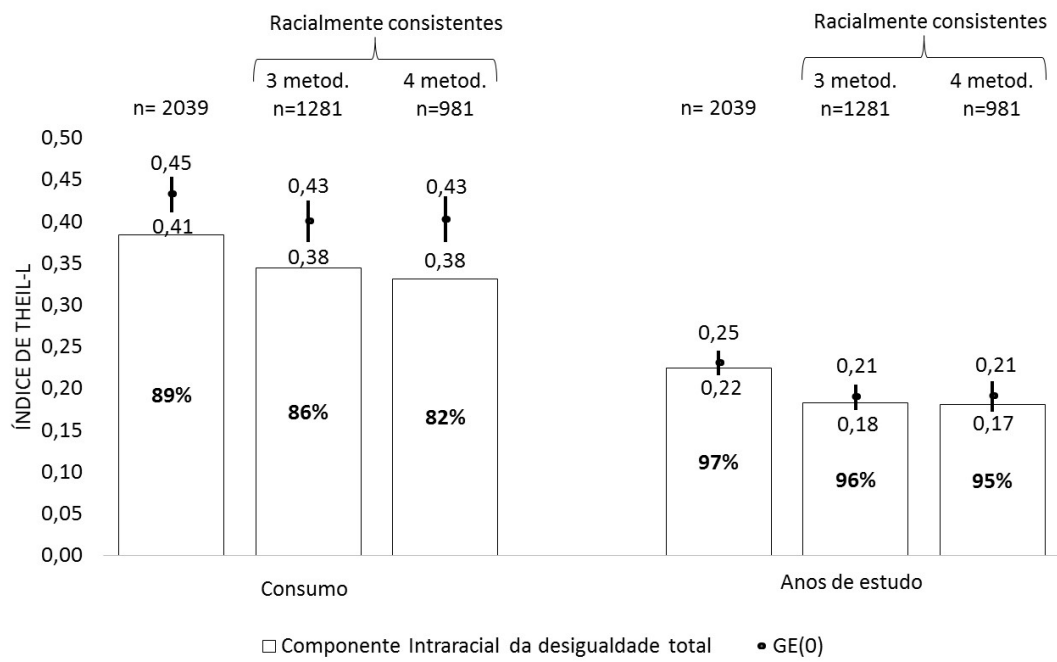

Nota: Os intervalos de $95 \%$ de confiança estatística baseiam-se em erros padrão estimados via procedimentos estatísticos do tipo bootstrap. O componente inter-racial é obtido a partir da diferença entre a estimativa pontual da desigualdade total e o seu componente intraracial. Todos os intervalos de confiança dos componentes intra e inter-raciais (ver anexo 3), tanto de consumo quanto de anos de estudo, apresentaram sobreposição.

Fonte: Elaboração própria a partir dos dados da PESB 2002.

Os inconsistentemente classificados, portanto, não alteram substantivamente as desigualdades inter-raciais de escolaridade e de consumo. Se não houvesse ambiguidades classificatórias no sistema utilizado pelo IBGE, a desigualdade entre grupos raciais não se alteraria de forma substantiva. Aparentemente, o sistema classificatório do IBGE diminui desigualdades inter-raciais em ambas as dimensões observadas, mas estas diferenças não são estatisticamente significativas.

\section{Conclusão}

Segundo o Censo Demográfico de 2010, mais de 40\% da população brasileira se autodenomina parda. Metade destes se consideraria branca e a outra metade preta caso fossem obrigados a escolher apenas entre estas duas opções (Bailey, 2008; Muniz, 2012). Esta é uma das razões pelas quais a categoria dos pardos é considerada ambígua e incerta, sendo denominada 
como a "válvula de escape" da negritude, a categoria social intermediária entre brancos e pretos (Degler, 1971).

De fato, ao se utilizarem quatro metodologias de classificação racial empregadas pela PESB 2002, cerca de metade dos pardos não se classificam (ou são classificados) como tais em todas elas. São os autodenominados pretos, entretanto, os que têm as maiores chances de serem racialmente inconsistentes. Esta inconsistência classificatória teria então alguma consequência sobre a desigualdade? A distribuição de anos de estudo e o consumo de bens e serviços seria afetada por esta inconsistência?

Nossa evidência mostra que os inconsistentemente classificados representam mais da metade das desigualdades totais, e cerca de um terço das desigualdades inter-raciais de escolaridade e consumo. Entretanto, apesar de quase metade da população ter sua raça/cor inconsistentemente classificada, as desigualdades percebidas de anos de estudo e consumo não se alteram substancialmente por causa disso. Quando simulamos estas desigualdades, sem considerar os casos inconsistentes em nossa amostra, a desigualdade educacional reduziu-se em $17 \%$ e a de consumo em $7 \%$, mostrando que a volatilidade racial classificatória do método de coleta utilizado afeta mais fortemente as desigualdades educacionais do que as de consumo.

As desigualdades inter-raciais, por outro lado, aumentam quando os casos inconsistentes são excluídos da análise. A participação do componente inter-racial da desigualdade educacional passa de $3 \%$ para $5 \%$, e de $11 \%$ para $18 \%$ no caso do consumo quando simulamos a desigualdade excluindo os casos racialmente inconsistentes. Estas variações relativas, entretanto, não são significativas ao nível de $95 \%$ de confiança estatística.

Em suma, por um lado os inconsistentemente classificados são responsáveis por uma parcela significativa das desigualdades totais e interraciais de escolaridade e consumo, mas, por outro, se os mesmos fossem desconsiderados do computo das desigualdades totais estas pouco se alterariam, apesar do aparente aumento relativo das desigualdades inter-raciais (Gráfico 5). A variabilidade da desigualdade diante da incerteza classificatória racial não deve, portanto, continuar a fazer parte da "postura obscurantista" (Feres Júnior, 2008, p. 72) daqueles que costumam utilizá-la como parte do argumento especulativo contra ações afirmativas baseadas em cotas raciais. Este artigo esclarece que em pelo menos duas dimensões da estratificação social entre as raças, educação e consumo, há evidência para se rechaçar a relevância da taxonomia para o nosso entendimento ou mensuração das desigualdades populacionais, ainda que, paradoxalmente, uma parcela considerável destas mesmas desigualdades seja devida àqueles cuja a cor é incerta. 


\section{Referências}

ALMEIDA, Alberto Carlos; SCHROEDER, Andréia; CHEIBUB, Zairo (orgs.). PESB: Pesquisa Social Brasileira, 2002 (Banco de dados). Rio de Janeiro: Universidade Federal Fluminense (UFF). In: Consórcio de Informações Sociais, $2004<$ http://www. cis.org.br> (3 jun. 2013).

AZEVEDO, Thales de. Cultura e situação racial no Brasil. Rio de Janeiro: Civilização Brasileira, 1966.

AZEVEDO, Thales de. As elites de cor numa cidade brasileira: um estudo de ascensão social e classes sociais e grupos de prestígio. Salvador: Ufba, 1996.

BAILEY, Stanley. Unmixing for race making in Brazil. American Journal of Sociology, v. 114, n. 03, p. 577-870, $2008<10.1086 / 592859>$.

BAILEY, Stanley; LOVEMAN, Mara; MUNIZ, Jerônimo. Measures of 'race' and the analysis of racial inequality in Brazil. Social Science Research, v. 42, n. 1, p. 106-119, $2013<10.1016 /$ j.ssresearch.2012.06.006>.

BAILEY, Stanley; TELLES, Edward. Multiracial versus collective black categories: examining census classification debates in Brazil. Ethnicities, v. 6, n. 1, p. 74-101, $2006<10.1177 / 1468796806061077>$.

CAMPOS, Luiz Augusto. 'We have a dream': cientistas sociais e a controvérsia sobre as cotas raciais na imprensa. Revista de Sociologia e Política, v. 20, n. 41, p. 53-73, $2012<10.1590 /$ S0104-44782012000100005>.

CARDOSO, Fernando Henrique. Negros em Florianópolis: relações sociais e econômicas. Florianópolis: Insular, 2000.

CARDOSO, Fernando Henrique. Capitalismo e escravidão no Brasil meridional: o negro na sociedade escravocrata do Rio Grande do Sul. Rio de Janeiro: Civilização Brasileira, 2003.

CARDOSO, Fernando Henrique; IANNI, Octavio. Cor e mobilidade social em Florianópolis: aspectos das relações entre negros e brancos numa comunidade do Brasil meridional. São Paulo: Companhia Editora Nacional, 1960.

COWELL, Frank. Measuring inequality. London: Prentice Hall/Harvester Wheatsheaf, 1995.

DA MATTA, Roberto. Carnavais, malandros e heróis: para uma sociologia do dilema brasileiro. Rio de Janeiro: Zahar, 1988.

DEGLER, Carl. Neither black nor white: slavery and race relations in Brazil and the United States. Madison: University of Wisconsin Press, 1971.

FERES JÚNIOR, João. Comparando justificações das políticas de ação afirmativa: Estados Unidos e Brasil. Estudos Afro-Asiáticos, v. 29, n. 1-3, p. 63-84, 2007.

FERES JÚNIOR, João. Ação afirmativa: política pública e opinião. Sinais Sociais, V. 3, n. 8, p. 38-77, 2008.

FERNANDES, Florestan. A integração dos negros na sociedade de classes. São Paulo: Dominus, 1965. 
FERNANDES, Florestan. O negro no mundo dos brancos. São Paulo: Global, 2007.

FERREIRA, Francisco; LANJOUW, Peter; NERI, Marcelo. A new poverty profile for Brazil using PPV, Pnad and census data. (Trabalho apresentado no 29을 Encontro da Associação Nacional de Pós-graduação em Economia, Anpec, 2001).

FERREIRA, Francisco; LEITE, Phillippe; LITCHFIELD, Julie. The rise and fall of Brazilian inequality, 1981-2004. Washington, D.C.: World Bank Development Research Group Poverty Team, 2006.

FILMER, D.; PRITCHETT, L. The effect of household wealth on educational attainment: evidence from 35 countries. Population and Development Review, v. 25, n. 1 , p. $85-120,1999<10.1111 /$ j. 1728-4457.1999.00085.x>.

FREYRE, Gilberto. Casa grande \& Senzala. 25. ed. Rio de Janeiro: José Olympio, 1987 [1933].

FRY, Peter; MAGGIE, Yvonne; MAIO, Marcos Chor; MONTEIRO, Simone; SANTOS, Ricardo Ventura (orgs.). Divisões perigosas: políticas raciais no Brasil contemporâneo. Rio de Janeiro: Editora Civilização Brasileira, 2007.

GUIMARÃES, Antônio. Entrevista com Carlos Hasenbalg. Tempo Social, v. 18, n. 2, p. 259-268, 2006.

GUIMARÃES, Antônio. Como trabalhar com "raça" em sociologia. Educação e Pesquisa, v. 29, n. 1, p. 93-107, 2003.

HARRIS, Marvin. Race relations in Minas Velhas, a community in the mountain region of central Brazil. In: Charles Wagley (org.). Race and class in rural Brazil. Paris: Unesco, 1952.

HASENBALG, Carlos. Discriminação e desigualdades raciais no Brasil. 2. ed. Belo Horizonte: Editora UFMG, 2005 [1979].

HASENBALG, Carlos; SILVA, Nelson do Valle. Educação e diferenças raciais na mobilidade ocupacional. In: Carlos Hasenbalg; Nelson do Valle Silva e Márcia Lima (orgs.). Cor e estratificação social. Rio de Janeiro: Contracapa, 1999.

HASENBALG, Carlos; SILVA, Nelson do Valle. Origens e destinos: desigualdades sociais ao longo da vida. Rio de Janeiro: Topbooks, 2003.

IANNI, Octavio. As metamorfoses do escravo. São Paulo: Difusão Europeia do Livro, 1962.

IANNI, Octavio. Raças e classes sociais no Brasil. 3. ed. São Paulo: Brasiliense, 1987.

IBGE. Características étnico-raciais da população: um estudo das categorias de classificação de cor ou raça, 2008. Rio de Janeiro: IBGE, Coordenação de população de indicadores sociais, 2011.

LOVEMAN, Mara. The race to progress: census-taking and nation making in Brazil. Hispanic Historical Review, v. 89, n. 3, p. 435-470, 2009.

LOVEMAN, Mara; MUNIZ, Jerônimo; BAILEY, Stanley. Brazil in black and white? Race categories, the census, and the study of inequality. Ethnic and Racial Studies, v. 35 , n. 8 , p. $1466-1483,2012<10.1080 / 01419870.2011 .607503>$. 
MAIO, Marcos Chor. O Projeto Unesco e a agenda das ciências sociais no Brasil dos anos 40 e 50. Revista Brasileira de Ciências Sociais, v. 14, n. 41, p. 141-158, 1999 $<10.1590 /$ S0102-69091999000300009>.

MCKENZIE, D. Measuring inequality with asset indicators. Journal of Population Economics, v. 18, n. 2, p. 229-260, 2005.

MOOKHERJEE, Dilip; SHORROCKS, Anthony. A decomposition analysis of the trend in UK income inequality. The Economic Journal, v. 92, n. 368, p. 886-902, 1982. $<10.2307 / 2232673>$

MORAES SILVA, Graziella. After racial democracy: contemporary puzzles in race relations in Brazil, Latin America and beyond from a boundaries perspective. Current Sociology. p. 1-19, $2015<10.1177 / 0011392115590488>$.

MUNANGA, Kabengele. Rediscutindo a mestiçagem no Brasil: identidade nacional versus identidade negra. Petrópolis: Vozes, 1999.

MUNIZ, Jerônimo O. Sobre o uso da variável raça-cor em estudos quantitativos. Rev. Sociol. Política, v. 18, n. 36, p. 277-291, 2010<10.1590/S0104-44782010000200017>.

MUNIZ, Jerônimo O. Preto no branco? Mensuração, relevância e concordância classificatória no país da incerteza racial. Dados, v. 55, n. 1, p. 251-282, 2012 $<10.1590 /$ S0011-52582012000100007>.

MUNIZ, Jerônimo O. Demographic dynamics of poverty and income inequality: the case of Brazil. Revista Brasileira de Estudos Populacionais, v. 29, n. 2, p. 323-348, 2012a $<10.1590 /$ S0102-30982012000200007>.

NERI, Marcelo C.; AMADEO, Edward J.; CARVALHO, Alexandre P.; NASCIMENTO, Mabel C.; DALTRINO, Manoel F.; RANGEL, Flávia D. Los activos, los mercados y la pobreza en Brasil. El Trimestre Económico, México, v. 66, n. 3, p. 419-458, 1999.

NOGUEIRA, Oracy. Tanto preto quanto branco: estudos de relações raciais. São Paulo: T.A. Queiroz, 1985.

NOGUEIRA, Oracy. Preconceito de marca: as relações raciais em Itapetininga. São Paulo: Edusp, 1998.

OLIVER, Melvin; SHAPIRO, Thomas. Black wealth, white wealth: a new perspective on racial inequality. New York: Routledge, 2006.

OSORIO, Rafael. Desigualdade racial e mobilidade social no Brasil: um balanço das teorias. In: Mário Theodoro (org.). As políticas públicas e a desigualdade racial no Brasil: 120 anos após a abolição. Brasília: Ipea, 2008. p. 65-96.

OSORIO, Rafael. A desigualdade racial de renda no Brasil: 1976-2006. UNB, 2009. (Tese de doutorado. Programa de Pós-Graduação em Sociologia)

PENA, Sérgio; BORTOLINI, Maria. Pode a genética definir quem deve se beneficiar das cotas universitárias e demais ações afirmativas? Estudos Avançados, v. 18, n. 50, p. 31-50, 2004.

PIERSON, Donald. Brancos e pretos na Bahia: estudo de contato racial. São Paulo: Companhia Editora Nacional, 1945. 
RAMOS, Lauro; VIEIRA, Maria. Determinantes da desigualdade de rendimentos no Brasil nos anos 90: discriminação, segmentação e heterogeneidade dos trabalhadores. In: Ricardo Henriques (org.). Desigualdade e pobreza no Brasil. Rio de Janeiro: Ipea, 2000. p. 159-176.

REDDY, Sanjay; POGGE, Thomas. How not to count the poor. In: Mimeograph, World Bank, 2002.

RIBEIRO, Carlos Antonio C. Classe, raça e mobilidade social no Brasil. Dados, v. 49, n. 4, p. 833-873, 2006.

ROCHA, Sonia. Estabelecimento e comparação de linhas de pobreza para o Brasil. Texto para Discussão do Ipea, n. 153. Rio de Janeiro, 1988.

ROCHA, Sonia. Opções metodológicas para a estimação de linhas de indigência e de pobreza no Brasil. Texto para Discussão do Ipea, n. 720. Rio de Janeiro, 2000.

SANSONE, L. Pai preto, filho negro: trabalho, cor e diferenças de gerações. Estudos Afro-Asiáticos, 25, 1993.

SILVA, Nelson do Valle. Black-white income differentials in Brazil, 1960. Michigan: Ann Arbor, 1978.

SILVA, Nelson do Valle. As duas faces da mobilidade. Dados, n. 21, p. 49-67, 1979.

SILVA, Nelson do Valle. O preço da cor: diferenciais raciais na distribuição de renda no Brasil. Pesquisa e planejamento econômico, v. 10, n. 1, p. 21-44, 1980.

SILVA, Nelson do Valle. Updating the cost of not being white in Brazil. In: PierreMichel Foutaine (org.). Race, class, and power in Brazil. Los Angeles, CA: University of California Press, 1985. p. 42-55.

SIMÕES, Solange; JERONYMO, Mauro. Quem é negro no Brasil? Identidade racial e sistemas de classificação em uma sociedade miscigenada. In: Neuma Aguiar (org.). Desigualdades sociais, redes de sociabilidade e participação política. Belo Horizonte: Editora UFMG, 2007. p. 119-138.

TELLES, Edward. Racismo à brasileira: uma nova perspectiva sociológica. Rio de Janeiro: Relume Dumará, 2003.

TELLES, Edward. Race in Another America: the significance of skin color in Brazil. New Jersey: Princeton University Press, 2004.

TELLES, Edward; LIM, Nelson. Does it matter who answers the race question? Racial classification and income inequality in Brazil. Demography, v. 35, n. 4, p. 465-474, 1998.

WAGLEY, Charles (org.). Race and class in rural Brazil. Paris: Unesco, 1952.

Autor correspondente:

Jeronimo Oliveira Muniz

Centro de Pesquisas Quantitativas em Ciências Sociais - CPEQS

Faculdade de Filosofia e Ciências Humanas - Fafich

Universidade Federal de Minas Gerais

Av. Antônio Carlos, 6627, Fafich 4194/4156 - Pampulha

31270-901 Belo Horizonte, MG, Brasil 
Anexo 1. Decomposição da desigualdade educacional por raça/cor

\begin{tabular}{|c|c|c|c|c|}
\hline & \multicolumn{4}{|c|}{ Anos de estudo } \\
\hline & Brancos & Pretos & Pardos & Inconsistentes \\
\hline \multicolumn{5}{|c|}{ Componente inta-racial da desigualdade total: $f_{j} \mathrm{GE}(0)_{j}$} \\
\hline $\mathrm{n}(1)$ & 0,0961 & 0,0370 & 0,0919 & \\
\hline$n(2)$ & 0,0417 & 0,0076 & 0,0378 & 0,1362 \\
\hline $\mathrm{n}(3)$ & 0,0579 & 0,0128 & 0,0444 & 0,1077 \\
\hline \multicolumn{5}{|c|}{ Componente inter-racial da desigualdade total: $f_{j} \ln \left(1 / \lambda_{j}\right)$} \\
\hline $\mathrm{n}(1)$ & $-0,0474$ & 0,0304 & 0,0234 & \\
\hline$n(2)$ & $-0,0520$ & 0,0050 & 0,0122 & 0,0428 \\
\hline$n(3)$ & $-0,0566$ & 0,0090 & 0,0094 & 0,0469 \\
\hline \multicolumn{5}{|c|}{ Distribuição relativa da população $\left(f_{j}=n_{j} / n\right)$} \\
\hline $\mathrm{n}(1)$ & 0,4855 & 0,1280 & 0,3865 & \\
\hline$n(2)$ & 0,2570 & 0,0319 & 0,1923 & 0,5189 \\
\hline$n(3)$ & 0,3413 & 0,0530 & 0,2339 & 0,3718 \\
\hline \multicolumn{5}{|c|}{ Desigualdade dentro da categoria: $\mathrm{GE}(0)_{\mathrm{j}}$} \\
\hline $\mathrm{n}(1)$ & 0,1979 & 0,2888 & 0,2379 & \\
\hline$n(2)$ & 0,1624 & 0,2386 & 0,1966 & 0,2624 \\
\hline$n(3)$ & 0,1695 & 0,2415 & 0,1898 & 0,2896 \\
\hline \multicolumn{5}{|l|}{ Renda relativa $\left(\lambda_{j}=\mu_{j} / \mu\right)$} \\
\hline$n(1)$ & 1,1025 & 0,7887 & 0,9412 & \\
\hline$n(2)$ & 1,2241 & 0,8541 & 0,9383 & 0,9208 \\
\hline$n(3)$ & 1,1803 & 0,8445 & 0,9605 & 0,8815 \\
\hline
\end{tabular}

n (1) = metodologia de coleta da informação racial utilizada pelo IBGE;

n (2) = metodologias de coleta da informação racial utilizada pela PESB;

n (3) = metodologias utilizadas pela PESB2002, exceto hipodescendência

Anexo 2. Decomposição de desigualdade de consumo por raça/cor

\begin{tabular}{|c|c|c|c|c|}
\hline & \multicolumn{4}{|c|}{ Consumo } \\
\hline & Brancos & Pretos & Pardos & Inconsistentes \\
\hline \multicolumn{5}{|c|}{ Componente inta-racial da desigualdade total: $f_{j} \mathrm{GE}(0)_{j}$} \\
\hline $\mathrm{n}(1)$ & 0,1770 & 0,0477 & 0,1600 & \\
\hline $\mathrm{n}(2)$ & 0,0715 & 0,0136 & 0,0745 & 0,2233 \\
\hline$n(3)$ & 0,1069 & 0,0196 & 0,0901 & 0,1685 \\
\hline \multicolumn{5}{|c|}{ Componente inter-racial da desigualdade total: $f_{j} \ln \left(1 / \lambda_{j}\right)$} \\
\hline $\mathrm{n}(1)$ & $-0,1291$ & 0,0685 & 0,1088 & \\
\hline$n(2)$ & $-0,1173$ & 0,0122 & 0,0586 & 0,0966 \\
\hline$n(3)$ & $-0,1254$ & 0,0219 & 0,0687 & 0,0825 \\
\hline \multicolumn{5}{|c|}{ Distribuição relativa da população $\left(f_{j}=n_{j} / n\right)$} \\
\hline $\mathrm{n}(1)$ & 0,4855 & 0,1280 & 0,3865 & \\
\hline$n(2)$ & 0,2570 & 0,0319 & 0,1923 & 0,5189 \\
\hline $\mathrm{n}(3)$ & 0,3413 & 0,0530 & 0,2339 & 0,3718 \\
\hline \multicolumn{5}{|c|}{ Desigualdade dentro da categoria: $\mathrm{GE}(0)_{\mathrm{j}}$} \\
\hline $\mathrm{n}(1)$ & 0,3646 & 0,3730 & 0,4139 & \\
\hline$n(2)$ & 0,2784 & 0,4281 & 0,3873 & 0,4304 \\
\hline$n(3)$ & 0,3133 & 0,3704 & 0,3853 & 0,4532 \\
\hline \multicolumn{5}{|l|}{ Consumo relativo $\left(\lambda_{j}=\mu_{j} / \mu\right)$} \\
\hline $\mathrm{n}(1)$ & 1,3045 & 0,5857 & 0,7547 & \\
\hline$n(2)$ & 1,5787 & 0,6823 & 0,7374 & 0,8302 \\
\hline$n(3)$ & 1,4439 & 0,6611 & 0,7455 & 0,8009 \\
\hline
\end{tabular}

$\mathrm{n}(1)=$ metodologia de coleta da informação racial utilizada pelo IBGE;

$\mathrm{n}(2)=$ metodologias de coleta da informação racial utilizada pela PESB;

n (3) = metodologias utilizadas pela PESB2002, exceto hipodescendência. 
Anexo 3. Intervalos de 95\% de confiança estatística para os componentes intra e inter-raciais das desigualdades de consumo e educação

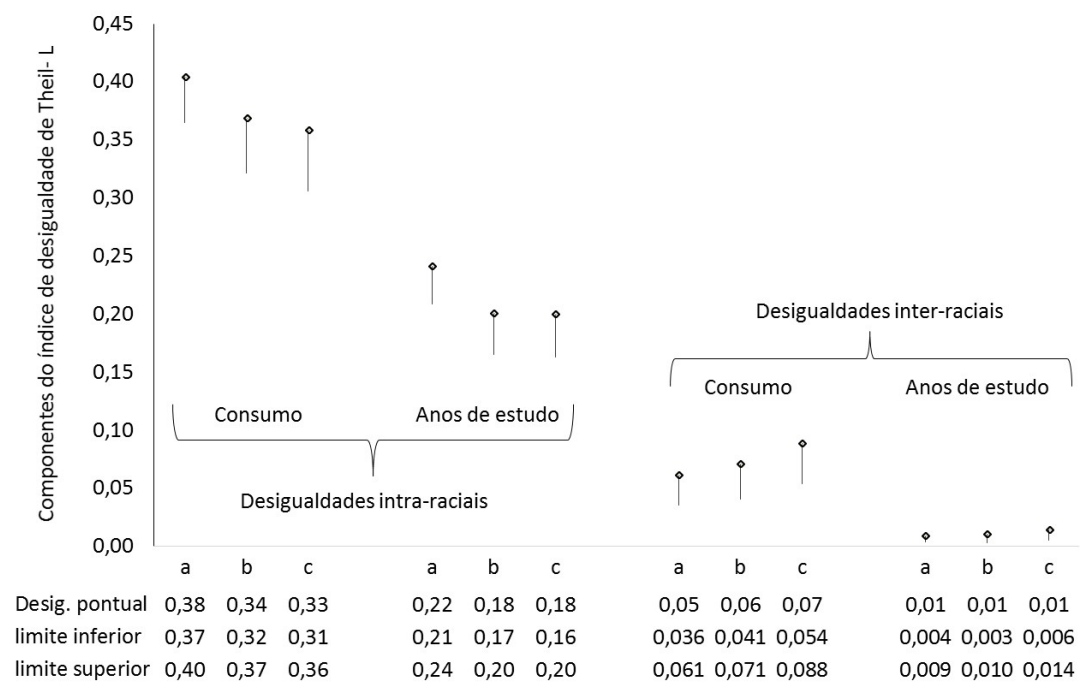

Nota: As letras no eixo horizontal indicam a quais amostras as medidas de desigualdade se referem: $\mathrm{a}=$ amostra analítica $(\mathrm{n}=2039) ; \mathrm{b}=$ amostra com casos racialmente consistentes em 3 metodologias $(\mathrm{n}=1281) ; \mathrm{c}=$ amostra com casos racialmente consistentes em 4 metodologias $(\mathrm{n}=981)$.

Fonte: Elaboração própria a partir dos dados da PESB 2002. 